\title{
ESTUDO POLÍNICO DE NOVE ESPÉCIES DA SUBTRIBO BACCHARINAE LESS. (ASTEREAE-ASTERACEAE), OCORRENTES NO SUL DO BRASIL ${ }^{1}$
}

\author{
PEDRO CESAR TEIXEIRADE SOUZA ${ }^{2}$ FABIANACARVALHO DE SOUZA ${ }^{3}$ \\ ANABELASILVEIRADE OLIVEIRA-DEBLE ${ }^{4}$ CLÁUDIABARBIERIFERREIRAMENDONÇA $^{5}$ \\ VANIAGONÇALVES-ESTEVES ${ }^{6}$
}

\section{RESUMO}

São palinologicamente estudadas cinco espécies de Baccharis L. (B. oblongifolia (Ruiz \& Pavón) Pers., $B$. leucocephala Dusén, B. longoattenuata A. S. de Oliveira, B. glutinosa Pers. e B. riograndensis Teodoro et Vidal), três espécies de Heterotholamus Less. (Heterothalamus alienus (Spreng.) O. Kuntze, H. psiadoides Less., H. rupestris Deble, Oliveira \& Marchiori) e uma espécie de Heterothalamulopsis Deble, Oliveira \& Marchiori (Heterothalamulopsis wagenitzii (F. Hellw.) Deble, Oliveira \& Marchiori). As amostras foram obtidas de material herborizado e os grãos de pólen foram acetolisados, medidos, descritos, analisados e fotomicrografados sob microscopia de luz; para os não acetolisados, usou-se microscopia eletrônica de varredura. Os grãos de pólen foram caracterizados quanto à forma, às dimensões, ao tipo da abertura, à ornamentação da superfície e à espessura da exina. Os resultados mostram que, para os três gêneros, os grãos de pólen são pequenos ou médios, isopolares, oblato-esferoidais ou prolato-esferoidais, tricolporados, com endoabertura lalongada, exina espinhosa e caveada. Próprios da tribo Astereae, não é possível a separação dos gêneros estudados, com base nos atributos palinológicos.

Palavras-chave: Asteraceae, Baccharinae, Baccharis, Heterothalamus, Heterothalamulopsis, pólen.

\section{ABSCTRACT}

[Palynologic study of nine Baccharinae Less. species (Astereae-Asteraceae) from southern Brasil]. Five species of Baccharis L. (Baccharis oblongifolia (Ruiz \& Pavón) Pers., B. leucocephala Dusén, B. longoattenuata A. S. de Oliveira, B. glutinosa Pers. e B. riograndensis Teodoro et Vidal), three species of Heterotholamus Less. (Heterothalamus alienus (Spreng.) O. Kuntze, H. psiadoides Less., H. rupestris Deble, Oliveira \& Marchiori) and one species of Heterothalamulopsis Deble, Oliveira \& Marchiori (Heterothalamulopsis wagenitzii (F. Hellw.) Deble, Oliveira \& Marchiori) were palinologically studied. The samples were obtained from herborized material, being the pollen acetolyzed, measured, described, analyzed and fotomicrographed under light microscope; the non-acetolyzed pollen grains were photomicrographed under scanning electron microscopy. The pollen were caracterized by the form, dimensions, types of aperture, surface ornaments and exine thickness. The results showed that all the three genera has small or medium, isopolar, oblate spheroidal or prolate spheroidal tricolporate pollen, with lalongate endoaperture, and spinose caveate exine. Typical from Astereae, it is not possible to separate the studied genera, based on palinological attributes.

Key words: Asteraceae, Baccharinae, Baccharis, Heterothalamus, Heterothalamulopsis, pollen.

1 Recebido em 14/08/2007 e aceito para publicação em 10/10/2007.

2 Graduando em Ciências Biológicas, Unigranrio, Técnico do Laboratório de Palinologia, Departamento de Botânica, Museu Nacional, Universidade Federal do Rio de Janeiro. Quinta da Boa Vista, São Cristóvão, Rio de Janeiro, RJ. CEP 20940-040.

3 Bióloga, Mestranda, Laboratório de Palinologia, Departamento de Botânica, Museu Nacional, Universidade Federal do Rio de Janeiro. Quinta da Boa Vista, São Cristóvão, Rio de Janeiro, RJ. CEP 20940-040.

${ }^{4}$ Bióloga MSc., bolsista CAPES, doutoranda do Programa de Pós-Graduação em Engenharia Florestal, Centro de Ciências Rurais, Universidade Federal de Santa Maria. CEP 97105-900, Santa Maria (RS). anabela.biol@gmail.com

5 Bióloga, Dra., Professora Visitante do Departamento de Botânica, Museu Nacional, Universidade Federal do Rio de Janeiro. Quinta da Boa Vista, São Cristóvão, Rio de Janeiro, RJ. CEP 20940-040.

${ }^{6}$ Bióloga, Dra., bolsista de Produtividade em Pesquisa do CNPq, Professora Associada do Departamento de Botânica, Museu Nacional, Universidade Federal do Rio de Janeiro. Quinta da Boa Vista, São Cristóvão, Rio de Janeiro, RJ. CEP 20940-040. 


\section{INTRODUÇÃO}

Em sua concepção tradicional, a subtribo Baccharinae Less. compreende quatro gêneros americanos: Archibaccharis Heering, Baccharidastrum Cabrera, Baccharis L. e Heterothalamus Less. (Giuliano, 2000). Redefinida por Nesom (1993, 1994), a subtribo passou a incluir sete gêneros da África (Commidendron DC., Melanodendron DC., Microglossa DC., Psiadia Jacq., Psiadiella Humbert, Sarcanthmum Cass., Vernoniopsis Humbert) e um do sudeste da Ásia (Heteroplexis C. C. Chang.).

Descrito por Deble et al. (2004), o gênero Heterothalamulopsis Deble, Oliveira \& Marchiori abriga uma espécie com características intermediárias entre Baccharis L. e Heterothalamus Lessing, o que requer avaliação mais ampla sobre seu posicionamento na subtribo Baccharinae Less.

As Baccharinae reúnem ervas, lianas, subarbustos, arbustos e árvores de folhas alternas, raro opostas, geralmente pontuado-glandulares e de margens inteiras ou serradas, sésseis ou pecioladas, concolores ou discolores, glabras ou com tricomas, de limbo desenvolvido ou reduzido a escamas inconspícuas (Nesom, 1994).

Neotropical, o gênero Baccharis L. (s.l.) compreende entre 400-500 espécies de ervas perenes, subarbustos, arbustos e pequenas árvores essencialmente dióicas, raramente monóicas, polígamo-dióicas ou imperfeitamente dióicas. No Brasil, ele está representado por aproximadamente 146 espécies, em sua maioria do sul e sudeste do país (Oliveira et al. 2006).

$\mathrm{O}$ gênero Heterothalamus $\mathrm{L}$ abriga três espécies de subarbustos e arbustos imperfeitamente dióicos, que se separam dos demais táxons da subtribo Baccharinae Lessing pelos capítulos masculinos com flores marginais neutras e liguladas, pelos capítulos femininos com páleas persistentes e flores carnosas, liguladas, bem como pelos aquênios glabros, angulosos, providos de 5-7-costas. A distribuição geográfica do gênero restringe-se ao sul do Brasil (Rio Gran- de do Sul e Santa Catarina), Uruguai e Argentina (Deble et al. 2005).

Monotípico e de caráter dióico, Heterothalamulopsis wagenitzii (F. Hellwig) Deble, Oliveira \& Marchiori apresenta capítulos femininos de receptáculo paleáceo, com flores de corola carnosa distintamente ligulada e aquênios cilíndricos, glabros, 10-costados. A espécie tem distribuição geográfica restrita aos Aparados da Serra Geral, no Rio Grande do Sul e Santa Catarina (Deble et al. 2005).

J. Müller (2006), em estudo sobre Baccharis L. e espécies relacionadas, colocou os gêneros Heterothalamus, Heterothalamulopsis e Baccharidastrum, reconhecidos por diversos autores como independentes, dentro do gênero Baccharis L., com base na estrutura da parede do aquênio, na forma das corolas e no ápice dos estigmas, mantendo apenas Archibaccharis como gênero independente.

No presente trabalho, optou-se por tratar os gêneros Baccharis, Heterothalamus e Heterothalamulopsis como independentes, fundamentando-se nas caraterísticas morfológicas dos mesmos. O estudo trata da análise dos grãos de pólen de Baccharis oblongifolia (Ruiz \& Pavón) Pers., B. leucocephala Dusén, $B$. longoattenuata A. S. de Oliveira., B. glutinosa Pers., B. riograndensis Teodoro et Vidal, Heterothalamus alienus (Spreng.) O. Kuntze, H. psiadoides Less., H. rupestris Deble, Oliveira \& Marchiori e Heterothalamulopsis wagenitzii (F. Hellw.) Deble, Oliveira \& Marchiori.

\section{MATERIAIS E MÉTODOS}

O material polínico foi obtido de exsicatas depositadas nos herbários PACA (Herbarium Anchieta, São Leopoldo/RS) e HDCF (Herbário do Departamento de Ciências Florestais, UFSM, Santa Maria/RS).

Para cada táxon determinou-se um espécime como "padrão", indicado por asterisco junto ao nome do coletor. Procurou-se estudar outros indivíduos de cada espécie, denominados "ma- 
terial de comparação", com a finalidade de se estabelecer a variação morfométrica entre espécimes. O material estudado é apresentado a seguir:

Baccharis oblongifolia (Ruiz \& Pavón) Pers. SC, Palhoça, *Reitz \& Klein n. 3.386, 09.VII.1956 (PACA 64.962); SC, Itajaí, morro da fazenda, Reitz \& Klein n. 1.905, 01.VII.1954 (PACA 64.950); K.P. Montenegro \& B. Rambo s.n., 11.XI.1949 (PACA 43.372); PR, Lapa, G. Hatschbach 152, 23.VII.1945 (PACA 30.239).

Baccharis leucocephala Dusén

RS, São José dos Ausentes, *L.P. Deble \& A.S. de Oliveira 5586, 05.XI.2004; RS, São José dos Ausentes, L.P. Deble \& A.S. de Oliveira 5597, 05.XI.2004; RS, São José dos Ausentes, L.P. Deble \& A.S. de Oliveira 5598, 05.XI.2004; RS, São José dos Ausentes, L.P. Deble \& A.S. de Oliveira 5599, 05.XI.2004.

Baccharis longoattenuata A. S. de Oliveira

RS, Caçapava do Sul, Guaritas, ${ }^{*}$ L.P. Deble \& A.S. de Oliveira 5581, 03.I.2004; RS, Caçapava do Sul, Guaritas, L.P. Deble \& A.S. de Oliveira 5591, 03.I.2004; RS, Caçapava do Sul, Guaritas, L.P. Deble \& A.S. de Oliveira 5592, 03.I.2004; RS, Caçapava do Sul, Guaritas, L. P. Deble \&A. S. de Oliveira 5590, 03.I.2004. Baccharis glutinosa Pers.

RS, Barra do Quaraí, Parque do Espinilho, *L.P. Deble \& A.S. de Oliveira 5600, 01.IV.2005; RS, Barra do Quaraí, Parque do Espinilho, L. P. Deble \& A. S. de Oliveira 5588, 01.IV.2005; RS, Barra do Quaraí, Parque do Espinilho, L. P. Deble \& A. S. de Oliveira 5601, 01.IV.2005; RS, Barra do Quaraí, Parque do Espinilho, L.P. Deble \& A.S. de Oliveira 5602, 01.IV.2005.

Baccharis riograndensis Teodoro et Vidal

RS, São Francisco de Assis, p. Manoel Viana, *L. P. Deble \& A. S. de Oliveira 5587, 01.IV.2004; RS, São Francisco de Assis, p. Manoel Viana, L. P. Deble \& A. S. de Oliveira 5595, 01.IV.2004; RS, São Francisco de Assis, p. Manoel Viana 5594, 3.IV.2004; RS, São Francisco de Assis, p. Manoel Viana, L. P. Deble \& A. S. de Oliveira 5596, 03.IV.2004.

Heterothalamulopsis wagenitzii (F.Hellw.) Deble, Oliveira \& Marchiori

RS, Cambará do Sul, *L.P. Deble \& A. S. de Oliveira 1518, 09.X.2003; RS, Cambará do Sul, L.P. Deble \& A. S. de Oliveira 1520, 11.X.2003; RS, Bom Jardim da Serra, L. P. Deble \&A. S. de Oliveira 1522, 05.XII.2003. Heterothalamus alienus (Spreng.) O. Kuntze
RS, São Joaquim, *L. P. Deble \& A. S. de Oliveira 5582, 06.XII.2003; RS, Santana da Boa Vista, p. Canguçu, L. P. Deble \& A. S. de Oliveira 1528, 05.XI.2004; RS, São Joaquim, L. P. Deble \& A. S. de Oliveira 5583, 06.XII.2003; Santa Catarina, Bom Jardim da Serra, L. P. Deble \& A. S. de Oliveira 1509, 06.XII.2003.

H. psiadoides Less.

RS, Santana da Boa Vista, *L.P. Deble \& A.S. de Oliveira 1513, 05.XI.2003; RS, Canguçu, L.P. Deble \& A.S. de Oliveira 1511, 05.XI.2003; RS, Cambará do Sul, L. P. Deble \& A. S. de Oliveira 1512, 12.XI.2003; RS, Cambará do Sul, L. P. Deble \& A. S. de Oliveira 1527, 12.XI.2003.

H. rupestris Deble, Oliveira \& Marchiori

RS, Bagé, Rincão do Inferno, ${ }^{*}$ L. P. Deble \& A. S. de Oliveira 1515, 27.VII.2003.

Para o estudo de microscopia de luz, o material polínico foi preparado segundo o método da acetólise, preconizado por Erdtman (1952), com modificações propostas em Melhem et al. (2003).

De cada espécime foram montadas pelo menos três lâminas permanentes com grãos de pólen acetolisados, dos quais foram tomadas as devidas mensurações, conforme Erdtman (1952), com algumas modificações propostas por Melhem et al. (2003).

Os grãos de pólen acetolisados foram medidos até sete dias após sua preparação, de acordo com Salgado-Labouriau (1973).

Do material padrão foram tomadas, aleatoriamente, 25 medidas de diâmetro polar (DP) e diâmetro equatorial (DE) dos grãos de pólen, em vista equatorial, e 10 medidas do diâmetro equatorial, em vista polar (DEVP) e o lado do apocolpo (LA), distribuídas em um mínimo de três lâminas (Salgado-Labouriau et al., 1965). Para os demais caracteres (aberturas, camadas da exina e diâmetros do material de comparação), foram mensurados aleatoriamente 10 grãos de pólen, distribuídos, no mínimo, em três lâminas e calculada a média aritmética.

No material padrão, o tratamento estatístico incluiu a média aritmética $(\mathrm{x})$, o desvio padrão 
da amostra (s), o desvio padrão da média $\left(\mathrm{s}_{\mathrm{x}}\right) \mathrm{e}$ o intervalo de confiança a $95 \%$ (IC 95\%).

A terminologia adotada foi a de Barth \& Melhem (1988) e de Punt et al. (2007), levando-se em consideração o tamanho, a forma, o número de aberturas e o padrão de ornamentação da sexina.

Para a obtenção das eletromicrografias, foram retiradas 2 ou 3 anteras das flores ou botões florais das referidas exsicatas, que foram maceradas com auxílio de pinça e estilete devidamente flambados, liberando os grãos de pólen (não acetolisados) sobre um suporte metálico previamente recoberto por fita adesiva de carbono dupla face. O material sobre o suporte foi metalizado em ouro puro, por cerca de três minutos. Levou-se o suporte para a observação em microscópio eletrônico de varredura (MEV) e o material polínico foi eletromicrografado em aparelho JSM-5310, pertencente ao Laboratório de Ultraestrutura Celular Hertha Meyer, do Instituto de Biofísica, da Universidade Federal do Rio de Janeiro.

\section{RESULTADOS}

As espécies de Baccharis (Figura A, 1-20) apresentaram grãos de pólen médios em sua maioria, e pequenos ou médios apenas em $B$. riograndensis, isopolares, prolato-esferoidais e oblato-esferoidais apenas em $B$. leucocephala (Tabelas 1,2), âmbito subtriangular, área polar pequena, 3-colporados, sexina espinhosa e caveada (Figura A1). Colpos longos, com membrana ornamentada, melhor visualizada sob MEV (Figura A 4, 8, 12, 16), endoaberturas nitidamente lalongadas (Tabela 3). Espinhos com ápice agudo e base columelada, com perfurações de tamanhos variados circundando a base (Figura $\mathrm{A}$ $8,10,12)$. Em B. leucocephala, os espinhos são mais longos e com base mais estreita (Figura A 5, 6). Os espinhos mais esparsos foram observados em B. riograndensis (Figura A 17, 18). As cáveas são estreitas $(0,3-0,5 \mu \mathrm{m}) \mathrm{em} B$. oblongifolia, $B$. glutinosa e $B$. leucocephala ou largas em B. longoattenuata (ca. 0,9 $\mu \mathrm{m}$ ) e (ca. 0,7 $\mu \mathrm{m}$ ) em B. riograndensis (Tabela 3).

O material de comparação (Tabela 4) apresentou grãos de pólen com forma e área polar semelhantes às do material padrão. Com relação aos valores dos diâmetros polar e equatorial, estes ficaram, em sua maioria, fora dos limites do intervalo de confiança, mas dentro dos limites da faixa de variação, quando comparados com o material padrão. No espécime padrão, Baccharis glutinosa apresentou grãos de pólen de tamanho médio e, nos de comparação, tamanho pequeno; em $B$. riograndensis, o espécime padrão apresentou grãos de pólen de tamanho pequeno e os de comparação (P. Deble \& A. S. de Oliveira 5595 e P. Deble \& A. S. de Oliveira 5596), tamanho médio.

Heterothalamulopsis wagenitzii (Figura A 21-23) apresentou grãos de pólen de tamanho pequeno, isopolares, prolato-esferoidais (Tabelas 1,2), âmbito subtriangular, área polar pequena, 3-colporados, sexina espinhosa e caveada. Colpos longos, com membrana ornamentada (Figura A, 24), endoaberturas nitidamente lalongadas (Tabela 3). Espinhos com ápice agudo e base columelada, com perfurações de tamanhos variados circundando a base (Figura A, 22). As cáveas são estreitas, de difícil visualização (ca. $0,4 \mu \mathrm{m}$ ).

O material de comparação (Tabela 4) apresentou grãos de pólen com forma e área polar semelhantes às do material padrão. Com relação aos valores dos diâmetros polar e equatorial, estes ficaram, na sua maioria, fora dos limites do intervalo de confiança, mas dentro dos limites da faixa de variação, quando comparados com o material padrão. No material padrão, Baccharis glutinosa apresentou grãos de pólen de tamanho médio e, nos de comparação, tamanho pequeno, ao passo que em $B$. riograndensis, o espécime padrão apresentou grãos de pólen de tamanho pequeno e os de comparação (P. Deble \& A. S. de Oliveira 5595 e P. Deble \& A. S. de Oliveira 5596), tamanho médio. 
Heterothalamulopsis wagenitzii (Figura A, 21-23) apresentou grãos de pólen de tamanho pequeno, isopolares, prolato-esferoidais (Tabelas 1,2), âmbito subtriangular, área polar pequena, 3-colporados, sexina espinhosa e caveada. Colpos longos, com membrana ornamentada (Figura A, 24), endoaberturas nitidamente lalongadas (Tabela 3). Espinhos com ápice agudo e base columelada, com perfurações de tamanhos variados circundando a base (Figura $\mathrm{A}, 22)$. As cáveas são estreitas, de difícil visualização (ca. $0,4 \mu \mathrm{m}$ ).

O material de comparação (Tabela 4) apresentou grãos de pólen com forma e área polar semelhantes ao material padrão. Com relação aos valores dos diâmetros polar e equatorial, estes ficaram, em sua maioria, fora dos limites do intervalo de confiança, mas dentro dos limites da faixa de variação, quando comparados com o material padrão.

Heterothalamus alienus, $H$. psiadoides e H. rupestris (Figura A, 25-36) apresentaram grãos de pólen pequenos, isopolares, prolatoesferoidais (Tabelas 1, 2), âmbito subtriangular, área polar grande apenas em $H$. psiadoides e pequena nas demais espécies, 3-colporados, sexina espinhosa e caveada (Figura A, 25, 30, 34). Colpos longos (Figura A, 23, 32, 36), endoaberturas nitidamente lalongadas (Tabela 3). Espinhos com ápice agudo e base columelada, com perfurações de tamanhos variados circundando a base (Figura A, 26, 29, 31). Em $H$. alienus, os espinhos são mais curtos e com base mais larga (Figura A, 25, 26). Espinhos com distribuição mais esparsa foram observados em $H$. rupestris (Figura A, 35, 36). As cáveas são estreitas (Figura A, 30, 34), de difícil visualização (Tabela 3).

O material de comparação (Tabela 4) apresentou grãos de pólen com forma e área polar semelhante ao material padrão. Com relação aos diâmetros polar e equatorial, estes ficaram dentro dos limites da faixa de variação e fora dos limites do intervalo de confiança, quando comparados com o material padrão.

\section{DISCUSSÃO E CONCLUSÃO}

As espécies de Baccharis L. apresentam grãos de pólen muito semelhantes, diferindo apenas quanto ao tamanho e forma. As dimensões dos espinhos variaram em $B$. leucocephala e, em relação à distância, os mais esparsos foram encontrados apenas em $B$. riograndensis.

Os grãos de pólen de Heterothalamulopsis e das espécies de Heterothalamus aqui analisadas são muito semelhantes entre si, bem como com os de Baccharis. As espécies de Heterothalamus apresentaram diferenças no tamanho da área polar e nas dimensões dos espinhos.

As primeiras referências sobre palinologia de Baccharis halimifolia L. (espécie-tipo) foram fornecidas por Wodehouse (1935) e Erdtman (1952); os mesmos autores definiram os grãos de pólen em Baccharis como oblato-esferoidais, pequenos até médios, com diâmetro polar de 18 $-32 \mu \mathrm{m}$ e diâmetro equatorial de $20-32 \mu \mathrm{m}$.

Stix (1960) criou o tipo Baccharis, com base em Baccharis heterophylla H.B.K., espécie centro-americana. As espécies sul-brasileiras dos gêneros Baccharis, Heterothalamus e Heterothalamulopsis apresentam características polínicas que permitem enquadrá-las no tipo descrito pela autora, por terem, igualmente, grãos de pólen pequenos a médios, oblato-esferoidais, tricolporados, endoabertura lalongada e sexina espiculada.

Espinar (1973) analisou o pólen de onze espécies centro-argentinas de Baccharis; destas, B. articulata Pers., B. crispa Spreng., B. pingraea DC. e B. stenophylla Ariza são também comuns à flora do Brasil. Para o conjunto das espécies, o pólen foi caracterizado como 3 colporado, endoaberturas lalongadas, exina de 3 $\mu \mathrm{m}$ de espessura, nexina delgada, sexina com teto fino, constituída por columelas delgadas, às vezes coalescentes, e espinhos de base larga, contraída subitamente até o ápice. As espécies aqui analisadas apresentaram a espessura da exina com valores maiores do que os encontra- 

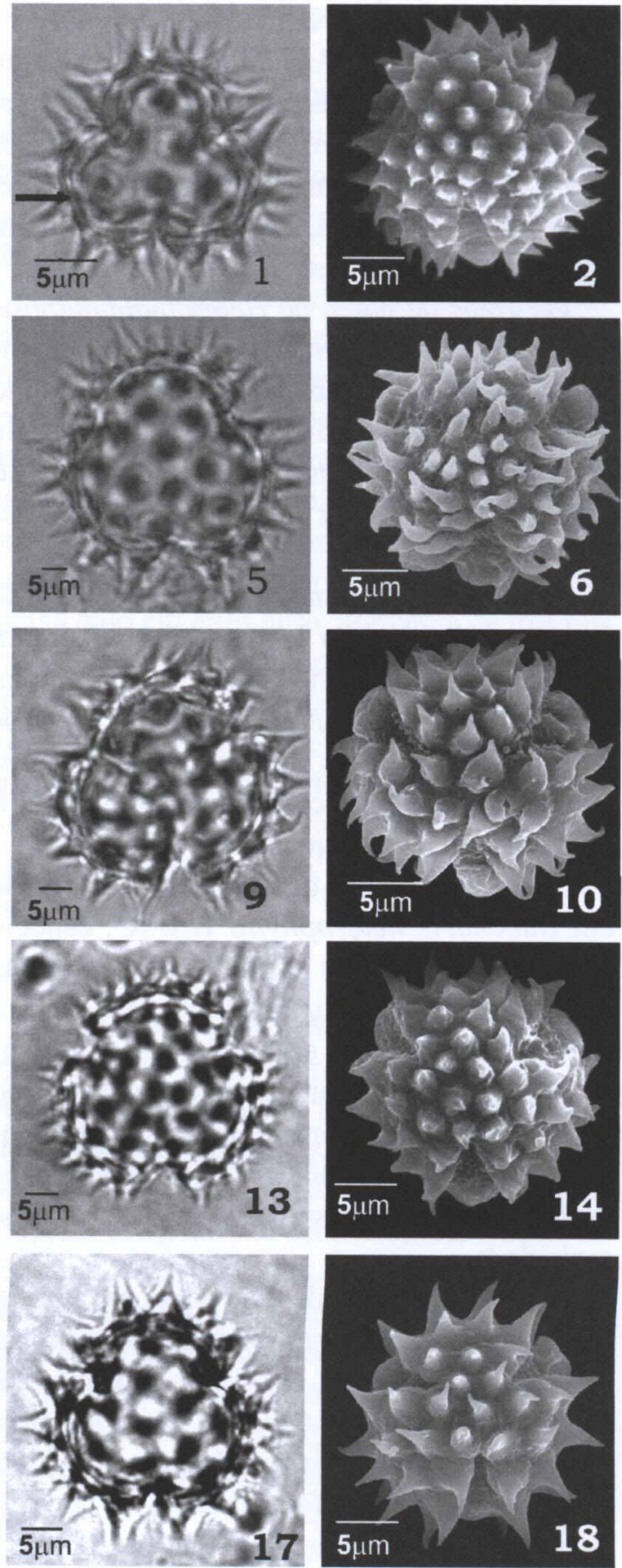
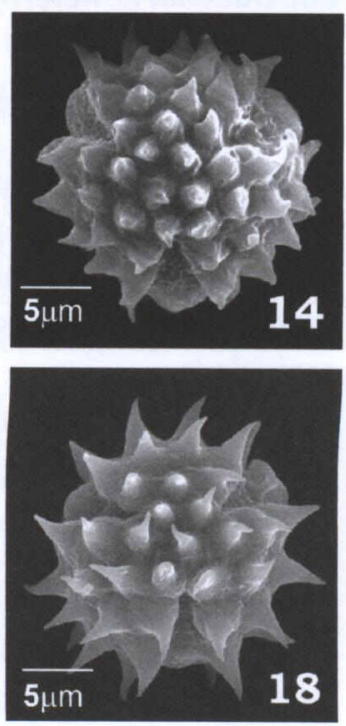

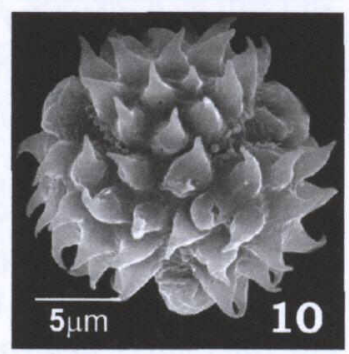

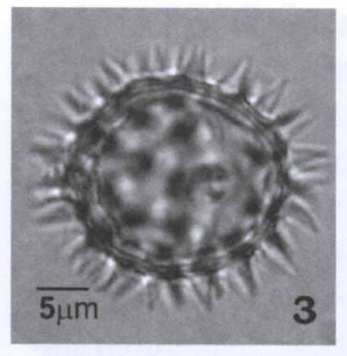
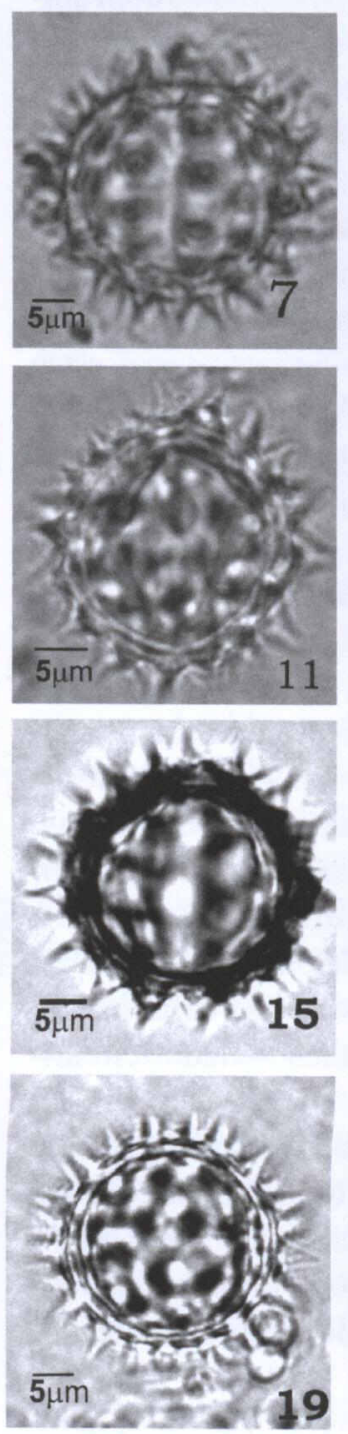
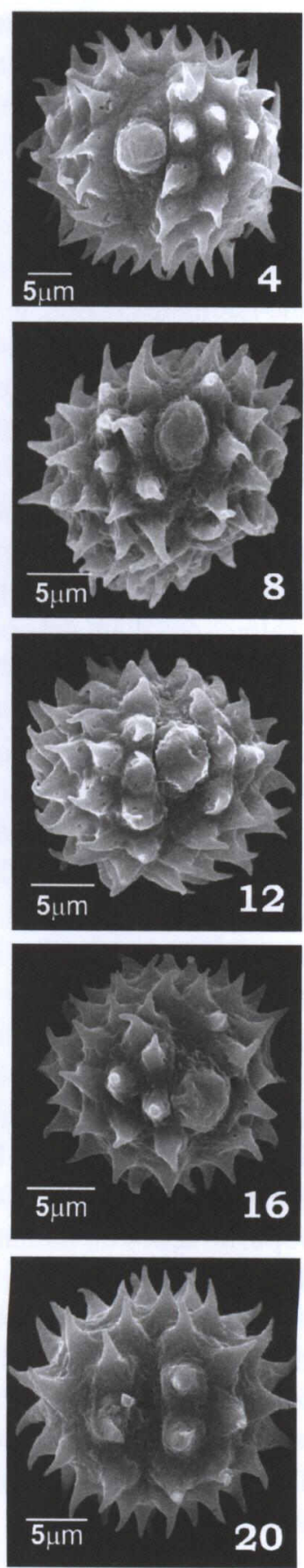

FIGURA A - Fotomicrografias e eletromicrografias do pólen de espécies de Baccharis L.: 1-4, B. oblongifolia - vista polar: corte óptico (1); vista geral na região do apocolpo MEV (2); vista equatorial: corte óptico e abertura (3); abertura e superfície MEV (4). 5-8, B. leucocephala - vista polar: corte óptico (5); vista geral na região do apocolpo MEV (6); vista equatorial: corte óptico e abertura (7); abertura e superfície MEV (8). 9-12, B. longoattenuata - vista polar: corte óptico (9); vista geral na região do apocolpo MEV (10); vista equatorial: corte óptico e abertura (11); abertura e superfície MEV (12). 13-16, B.glutinosa - vista polar: corte óptico (13); vista geral na região do apocolpo MEV (14); vista equatorial: corte óptico e abertura (15); abertura e superficie MEV (16). 17-20, B. riograndensis - vista polar: corte óptico (17); vista geral na região do apocolpo MEV (18); vista equatorial: corte óptico e abertura (19); abertura e superfície MEV (20). As setas indicam a cávea. 

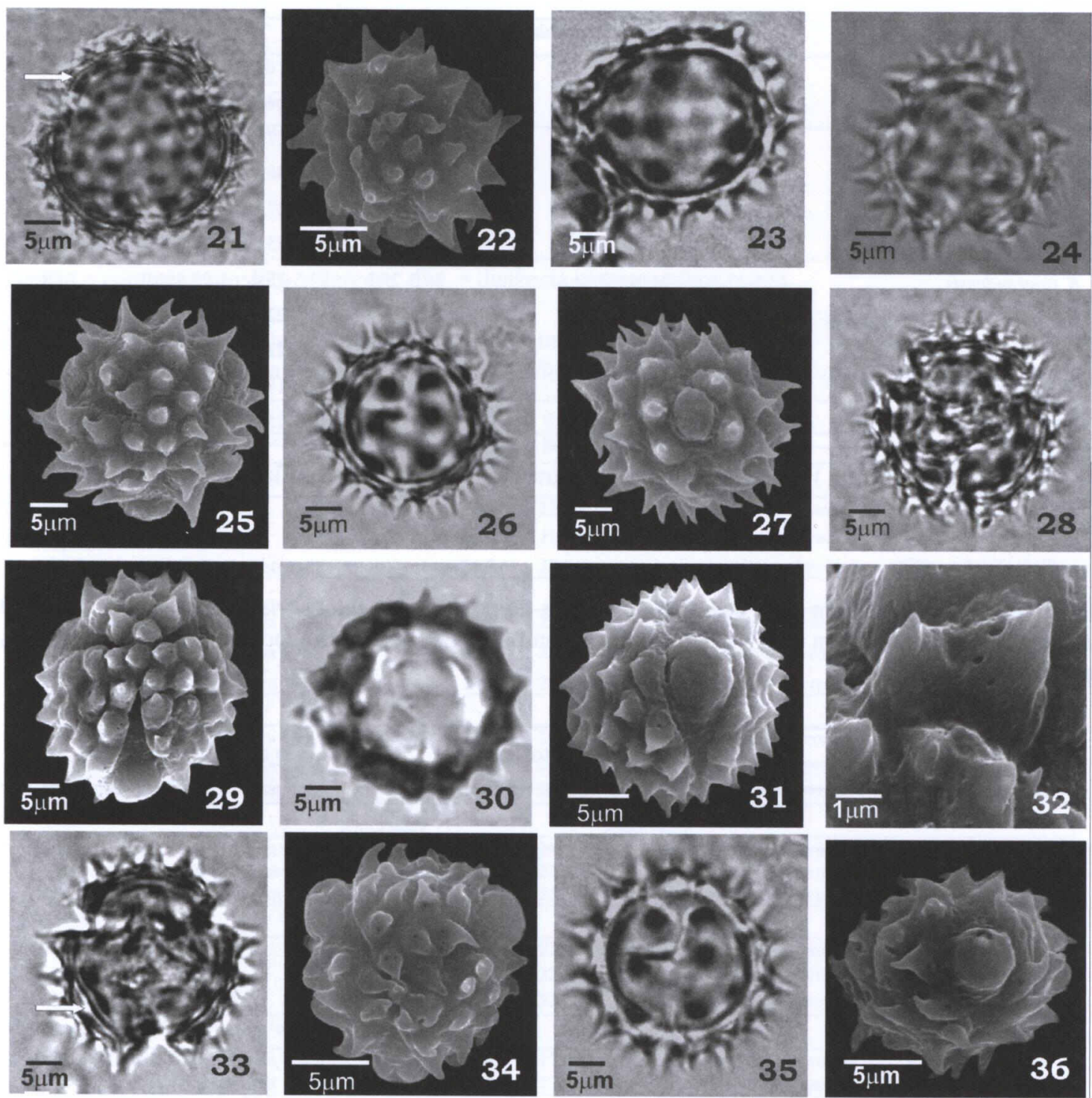

FIGURA A (continuação) - Fotomicrografias e eletromicrografias de Heterothalamulopsis e de Heterothalamus: 21-23, Heterothalamulopsis wagenitzii - vista polar: corte óptico (21); vista geral na região do apocolpo MEV (22); vista equatorial: corte óptico e abertura (23). 24-27, Heterothalamus alienus - vista polar: corte óptico (24); vista geral na região do apocolpo MEV (25); vista equatorial: corte óptico e abertura (26); abertura e superfície MEV (27). 28-32, $H$. psiadoides - vista polar: corte óptico (28); vista geral na região do apocolpo MEV (29); vista equatorial: abertura (30); abertura MEV (31); superfície MEV (32). 33-36, H. rupestris - vista polar: corte óptico (33); vista geral na região do apocolpo MEV (34); vista equatorial: corte óptico e abertura (35); abertura e superfície (36). As setas indicam a cávea. 
TABELA 1. Medidas (em $\mu \mathrm{m}$ ) dos grãos de pólen de espécies de Baccharis, Heterothalamulopsis e Heterothalamus ( $\mathrm{n}=25)$, em vista equatorial: diâmetro polar (DP); diâmetro equatorial (DE).

\begin{tabular}{lcccccc}
\hline \multicolumn{1}{c}{ Espécies } & \multicolumn{3}{c}{ Diâmetro polar } & \multicolumn{3}{c}{ Diâmetro equatorial } \\
\hline & Faixa de & $\overline{\mathrm{x}} \pm \mathrm{S} \overline{\mathrm{x}}$ & IC $95 \%$ & Faixa de & $\overline{\mathrm{x}} \pm \mathrm{S} \overline{\mathrm{x}}$ & $\mathrm{P} / \mathrm{E}$ \\
& variação & & & variação & & \\
\hline B. oblongifolia & $22,5-30,0$ & $26,1 \pm 0,4$ & $25,3-26,9$ & $20,0-27,5$ & $23,4 \pm 0,4$ & 1,11 \\
B. leucocephala & $26,2-27,5$ & $27,1 \pm 0,1$ & $27,0-27,2$ & $28,7-30,0$ & $29,7 \pm 0,1$ & 0,91 \\
B. longoattenuata & $27,5-32,5$ & $30,0 \pm 0,1$ & $29,7-30,3$ & $25,0-27,5$ & $27,4 \pm 0,1$ & 1,09 \\
B. glutinosa & $25,0-27,5$ & $26,1 \pm 0,2$ & $25,6-26,6$ & $22,5-25,0$ & $23,6 \pm 0,2$ & 1,10 \\
B. riograndensis & $22,5-27,5$ & $24,9 \pm 0,2$ & $24,5-25,3$ & $22,5-25,0$ & $22,7 \pm 0,1$ & 1,09 \\
Heterothalamulopsis wagenitzii & $20,0-22,5$ & $21,0 \pm 0,2$ & $20,5-21,5$ & $17,5-22,5$ & $18,6 \pm 0,3$ & 1,12 \\
Heterothalamus alienus & $20,0-25,0$ & $22,6 \pm 0,2$ & $22,2-22,8$ & $17,5-22,5$ & $20,8 \pm 0,3$ & 1,08 \\
H. psiadoides & $17,5-22,5$ & $19,5 \pm 0,2$ & $18,9-19,7$ & $15,0-18,7$ & $17,5 \pm 0,2$ & 1,11 \\
H. rupestris & $22,5-27,5$ & $23,8 \pm 0,3$ & $23,2-24,4$ & $17,5-25,0$ & $21,6 \pm 0,4$ & 1,10 \\
\hline
\end{tabular}

$(\overline{\mathrm{x}})=$ média aritmética; $(\mathrm{S} \overline{\mathrm{x}})=$ desvio padrão da amostra; $(\mathrm{IC})=$ intervalo de confiança .

TABELA 2. Medidas (em $\mu \mathrm{m}$ ) dos grãos de pólen de espécies de Baccharis, Heterothalamulopsis e Heterothalamus $(\mathrm{n}=10)$, em vista polar: diâmetro equatorial (DEVP); lado do apocolpo (LA) e índice da área polar (IAP).

\begin{tabular}{lccccc}
\hline \multirow{2}{*}{ Espécies } & \multicolumn{2}{c}{ Diâmetro equatorial } & \multicolumn{3}{c}{ Lado do apocolpo } \\
& $\begin{array}{l}\text { Faixa de } \\
\text { Variação }\end{array}$ & $\overline{\mathrm{X}}$ & $\begin{array}{c}\text { Faixa de } \\
\text { Variação }\end{array}$ & $\overline{\mathrm{X}}$ & IAP \\
\hline B. oblongifolia & $22,5-25,0$ & 22,7 & $8,7-10,0$ & 9,7 & 0,43 \\
B. leucocephala & $27,5-30,0$ & 29,2 & $6,25-7,5$ & 7,2 & 0,25 \\
B. longoattenuata & $30,0-32,5$ & 31,7 & $2,5-7,5$ & 8,0 & 0,25 \\
B. glutinosa & $27,5-28,7$ & 27,7 & $7,5-8,75$ & 7,7 & 0,28 \\
B. riograndensis & $22,5-23,7$ & 22,7 & $7,5-8,7$ & 8,0 & 0,35 \\
Heterothalamulopsis wagenitzii & $20,0-22,5$ & 20,5 & $8,0-9,2$ & 8,7 & 0,42 \\
Heterothalamus alienus & $20,0-22,5$ & 21,0 & $7,5-10,0$ & 9,4 & 0,45 \\
H. psiadoides & $16,2-18,7$ & 17,5 & $8,7-11,2$ & 9,6 & 0,55 \\
H. rupestris & $17,5-22,5$ & 18,2 & $7,5-9,5$ & 8,2 & 0,45 \\
\hline
\end{tabular}

$(\overline{\mathrm{x}})=$ média aritmética 
TABELA 3. Médias (em $\mu \mathrm{m}$ ) das medidas das aberturas, das camadas de exina e dos espinhos dos grãos de pólen, para as espécies estudadas de Baccharis, Heterothalamulopsis e Heterothalamus $(\mathrm{n}=10)$.

\begin{tabular}{|c|c|c|c|c|c|c|c|c|c|c|c|}
\hline \multirow[t]{2}{*}{ Espécies } & \multicolumn{2}{|c|}{ Colpo } & \multicolumn{2}{|c|}{ Endoabertura } & \multicolumn{4}{|c|}{ Espessura da exina } & \multicolumn{3}{|c|}{ Espinhos } \\
\hline & compr. & larg. & compr. & larg. & exina & sexina & nexina & cava & compr. & larg. & dist. \\
\hline B. oblongifolia & 15,0 & 3,0 & 3,5 & 5,5 & 5,0 & 4,0 & 0,7 & 0,3 & 3,0 & 1,0 & 3,5 \\
\hline B. leucocephala & 17,5 & 4,5 & 4,3 & 9,5 & 7,0 & 5,5 & 1,0 & 0,5 & 4,0 & 0,8 & 6,7 \\
\hline B. longoattenuata & 16,4 & 3,5 & 4,4 & 8,7 & 6,7 & 4,9 & 0,9 & 0,9 & 3,5 & 1,0 & 6,5 \\
\hline B. glutinosa & 16,0 & 2,7 & 3,0 & 5,0 & 5,3 & 4,3 & 0,7 & 0,3 & 3,3 & 1,0 & 5,0 \\
\hline B. riograndensis & 15,5 & 1,4 & 1,5 & 7,4 & 6,3 & 4,8 & 0,8 & 0,7 & 3,6 & 1,0 & 7,0 \\
\hline Heterothalamulopsis wagenitzii & 12,2 & 2,0 & 4,0 & 6,3 & 4,8 & 3,8 & 0,6 & 0,4 & 2,8 & 1,0 & 5,0 \\
\hline Heterothalamus alienus & 12,8 & 2,2 & 3,0 & 4,8 & 4,4 & 3,5 & 0,6 & 0,3 & 2,5 & 1,2 & 4,0 \\
\hline H. psiadoides & 12,4 & 2,0 & 4,7 & 6,5 & 5,0 & 4,0 & 0,6 & 0,4 & 3,0 & 1,0 & 5,0 \\
\hline H. rupestris & 13,7 & 2,7 & 3,5 & 5,2 & 5,0 & 4,0 & 0,7 & 0,3 & 3,0 & 1,0 & 6,0 \\
\hline
\end{tabular}

compr. $=$ comprimento; larg. $=$ largura; dist. $=$ distância entre espinhos.

TABELA 4. Medidas (em $\mu \mathrm{m})$ dos grãos de pólen do material de comparação $(\mathrm{n}=10)$, relação DP/DE e forma dos grãos de pólen, para as espécies estudadas de Baccharis, Heterothalamulopsis e Heterothalamus.

\begin{tabular}{|c|c|c|c|c|c|c|c|}
\hline \multirow[t]{2}{*}{ Espécie } & \multicolumn{3}{|c|}{ Vista equatorial } & & \multicolumn{3}{|c|}{ Vista polar } \\
\hline & DP & $\mathrm{DE}$ & DP/DE & Forma & DEVP & LA & IAP \\
\hline \multicolumn{8}{|l|}{ B. oblongifolia } \\
\hline G. Hatschbach 152 & 25,2 & 22,0 & 1,14 & prolato-esferoidal & 25,2 & 8,4 & 0,34 \\
\hline Reitz \& Klein n.1905 & 25,0 & 21,7 & 1,15 & prolato-esferoidal & 24,9 & 9,7 & 0,38 \\
\hline K.P. Montenegro \& B. Rambo s.n. & 25,3 & 22,3 & 1,13 & prolato-esferoidal & 25,6 & 8,2 & 0,32 \\
\hline \multicolumn{8}{|l|}{ B.glutinosa } \\
\hline L.P. Deble \& A.S. de Oliveira 5601 & 24,7 & 22,5 & 1,10 & prolato-esferoidal & 26,7 & 8,0 & 0,30 \\
\hline L.P. Deble \& A.S. de Oliveira 5588 & 24,8 & 22,7 & 1,09 & prolato-esferoidal & 26,9 & 8,7 & 0,32 \\
\hline L.P. Deble \& A.S. de Oliveira 5602 & 24,7 & 22,6 & 1,09 & prolato-esferoidal & 26,8 & 8,5 & 0,32 \\
\hline \multicolumn{8}{|l|}{ B. Leucocephala } \\
\hline L.P. Deble \& A.S. de Oliveira 5597 & 27,1 & 29,7 & 0,91 & oblato-esferoidal & 29,2 & 7,2 & 0,25 \\
\hline L.P. Deble \& A.S. de Oliveira 5598 & 27,3 & 29,9 & 0,91 & oblato-esferoidal & 29,8 & 7,6 & 0,25 \\
\hline L.P. Deble \& A.S. de Oliveira 5599 & 26,9 & 29,3 & 0,91 & oblato-esferoidal & 29,5 & 7,5 & 0,25 \\
\hline \multicolumn{8}{|l|}{ B. longoattenuata } \\
\hline L.P. Deble \& A.S. de Oliveira 5590 & 29,5 & 26,5 & 1,11 & prolato-esferoidal & 31,5 & 7,3 & 0,23 \\
\hline L.P. Deble \& A.S. de Oliveira 5592 & 29,8 & 27,0 & 1,10 & prolato-esferoidal & 31,7 & 7,5 & 0,24 \\
\hline L.P. Deble \& A.S. de Oliveira 5591 & 28,7 & 26,1 & 1,10 & prolato-esferoidal & 30,8 & 6,9 & 0,22 \\
\hline \multicolumn{8}{|l|}{ B. riograndensis } \\
\hline Manoel Viana 5594 & 24,5 & 22,0 & 1,11 & prolato-esferoidal & 25,5 & 7,9 & 0,31 \\
\hline Manoel Viana 5595 & 25,0 & 22,5 & 1,12 & prolato-esferoidal & 25,3 & 7,8 & 0,31 \\
\hline Manoel Viana 5596 & 26,0 & 23,2 & 1,12 & prolato-esferoidal & 25,2 & 7,7 & 0,30 \\
\hline \multicolumn{8}{|l|}{ Heterothalamulopsis wagenitzii } \\
\hline L.P. Deble \& A.S. de Oliveira 1522 & 20,5 & 17,9 & 1,14 & prolato-esferoidal & 19,3 & 7,3 & 0,38 \\
\hline L.P. Deble \& A.S. de Oliveira 1520 & 20,0 & 17,7 & 1,12 & prolato-esferoidal & 19,0 & 7,0 & 0,37 \\
\hline \multicolumn{8}{|l|}{ Heterothalamus alienus } \\
\hline L.P. Deble \& A.S. de Oliveira 1528 & 23,7 & 20,0 & 1,18 & prolato-esferoidal & 21,2 & 7,5 & 0,35 \\
\hline L.P. Deble \& A.S. de Oliveira 5583 & 24,0 & 20,4 & 1,18 & prolato-esferoidal & 21,2 & 6,8 & 0,32 \\
\hline L.P. Deble \& A.S. de Oliveira 1509 & 23,9 & 20,2 & 1,18 & prolato-esferoidal & 21,3 & 7,2 & 0,34 \\
\hline \multicolumn{8}{|l|}{ H. psiadoides } \\
\hline L.P. Deble \& A.S. de Oliveira 1527 & 20,2 & 18,7 & 1,08 & prolato-esferoidal & 21,5 & 8,7 & 0,40 \\
\hline L.P. Deble \& A.S. de Oliveira 1512 & 21,2 & 19,1 & 1,11 & prolato-esferoidal & 19,7 & 7,6 & 0,38 \\
\hline L.P. Deble \& A.S. de Oliveira 1511 & 20,8 & 18,9 & 1,10 & prolato-esferoidal & 19,8 & 9,5 & 0,48 \\
\hline
\end{tabular}

$\mathrm{P}=$ diâmetro polar; $\mathrm{E}=$ Diâmetro equatorial; $\mathrm{DEVP}=$ diâmetro equatorial em vista polar; $\mathrm{LA}=$ lado do apocolpo; $\mathrm{IAP}=$ índice da área polar 
dos pelo autor e espinhos nem sempre com as características acima apontadas.

A homogeneidade da tribo Astereae foi observada por Nair \& Lawrence (1985). As autoras caracterizaram a tribo como tendo grãos de pólen 3-colporados, com endoabertura lalongada, exina espinhosa e presença de cávea. Os gêneros aqui analisados foram considerados estenopalinológicos e, portanto, pode-se concordar com a afirmativa dos autores.

Barth (1989) analisou amostras de mel brasileiro contendo grãos de pólen não acetolisados. O tipo Compositae B, criado pela autora, reuniu Baccharidastrum sp. e Baccharis calvescens DC. Este tipo (Compositae B) foi caracterizado por apresentar grãos de pólen oblato-esferoidais, 3-colporados, com colpos longos, endoabertura circular, apocolpos pequenos, ausência de cávea e espículos agudos. Confrontando as informações de Barth (1989) com as descrições aqui apresentadas, conclui-se que as espécies em estudo não podem ser enquadradas no tipo B, principalmente pela presença de cávea.

Na descrição de Baccharis trinervis (Lam.) Pers., Roubik \& Moreno (1991) reconheceram grãos de pólen prolato-esferoidais, 3-colporados, com endoaberturas lalongadas e sexina espinho$\mathrm{sa}$, características igualmente observadas pelos autores no presente trabalho, com exceção da presença da cávea, não citada pelos autores.

Mendonça et al. (2002) descreveram palinologicamente três espécies de Baccharis (B. archtostaphylloides Baker, B. serrulata Pers. e B. singularis (Vell.) Barroso), caracterizando-as por apresentar grãos de pólen pequenos ou médios, oblato-esferoidais, de área polar muito pequena, 3-colporados, com exina espinhosa e caveada. Confrontando as descrições observadas no presente trabalho, pode-se concluir que as espécies apresentaram características polínicas semelhantes.

Em estudo da variabilidade polínica em plantas de Campos de Jordão, Melhem et al. (2003) descreveram seis espécies de Baccharis (B. anomala DC., B. dracunculifolia DC., $B$. microdonta DC., B. pentziifolia Sch.-Bip. ex Baker, B. sessiliflora Vahl e B. trimera (Less.) DC.). Apesar de distintas das presentemente estudadas, tais espécies apresentaram características semelhantes.

Como conclusão, pode-se afirmar que as espécies dos três gêneros analisados possuem características muito parecidas entre si e já mencionadas para a tribo Astereae, não sendo possível separá-las com base nos atributos palinológicos.

\section{BIBLIOGRAFIA}

Barth, O.M., Melhem, T.S. Glossário ilustrado de Palinologia, Campinas: Ed. Unicamp, 1988, 76p., 101 figs.

Barth, O.M. O pólen no mel brasileiro. Rio de Janeiro: Gráfica Luxor, 1989. 151p.

Deble, L. P., Oliveira, A. S. de, Marchiori, J. N. C. Heterothalamulopsis, gênero novo da subtribo Baccharinae Lessing (Astereae-Asteraceae). Ciência Florestal, Santa Maria, v. 14, n. 1, p. 1-7, 2004.

Deble, L. P., Oliveira, A. S. de, Marchiori, J. N. C. O gênero Heterothalamus Lessing e táxones afins. Balduinia, Santa Maria, n. 1, p. 1-20, 2005.

Erdtman, G. Pollen morphology and plant taxonomy Angiosperms. I-XII Stockholm: Almqvisit \& Wiksel, 1952.539p.

Espinar, L. A. Las espécies de Baccharis (Compositae) de Argentina Central. Bol. Acad. Nac. Cienc. Cordoba, v. 50, n. 1-4, p. 174-305, 1973.

Giuliano, D. A. Subtribu Baccharinae. In: Hunziker, A. T. Flora Fanerogámica Argentina, Córdoba, 2000. v. 66.73p.

Melhem, T.S., Cruz-Barros, M.A. V., Corrêa, A. M.S., Makino-Watanabe, H., Silvestre-Capelato, M.S.F., Gonçalves-Esteves, V. Variabilidade polínica em plantas de Campos de Jordão (São Paulo, Brasil). Boletim do Instituto de Botânica, v. 16, p. 9-104, 2003.

Mendonça, C.B.F., Gonçalves-Esteves, V., Esteves, R. Palinologia de espécies de Asteroideae (Compositae), ocorrentes na restinga de Carapebus, Carapebus, Rio de Janeiro. Hoehnea, v. 29, n. 3, p. 233-240, 2002. 
Müller, J. Systematics of Baccharis (CompositaeAstereae) in Bolivia, including an overview of the genus. Systematics Botany Monographs, v. 76, p. 1-339, 2006.

Nair, P. K. K., Lawrence, R. Advances in Pollen-Spore. Índia: Scholar Publishing House, 1985. v.14.p. 176.

Nesom, G. Aztecaster (Asteraceae: Astereae), a new ditypic genus of dioecious shrubs from Mexico, with redefinitions of the subtribes Hinterhuberinae and Baccharidinae. Phytologia, Huntsville, v. 75, n. 1, p. 62, 1993.

Nesom, G. Subtribal classification of the Astereae (Asteraceae). Phytologia, Huntsville, v. 76, p. 193-274, 1994.

Oliveira, A. S. de, Deble, L. P., Schneider, A. A., Marchiori, J. N. C. Cheklist do gênero Baccharis L. para o Brasil (Asteraceae-Astereae). Balduinia, Santa Maria, n. 9, p. 17-27, 2006.

Oliveira, A. S. de, Deble, L.P. Duas novas espécies sulbrasileiras de Baccharis L. (Asteraceae-Astereae). Balduinia, Santa Maria, n. 9, p. 4-9, 2006.
Punt, W., Blackmore, S., Nilsson, S., Le Thomas, A. Glossary of pollen and spore terminology. Review of Paleobotany and Palynology, v. 143, p. 1-81, 2007.

Roubik, D.W., Moreno, P.J.E. Pollen and spores of Barro Colorado Island. New York: Missouri Botanical Garden, 1991.

Salgado-Labouriau, M.L. Contribuição à Palinologia dos Cerrados. Academia Brasileira de Ciências, Rio de Janeiro, 1973.

Salgado-Labouriau, M. L., Vanzolini, P. E., Melhem, T. S. Variation of polar axes and equatorial diameters in pollen grains of two species of Cassia. Grana Palynologica, Stockholm, v.6, p.166-176, 1965.

Stix, E. Pollenmorpholosgische untersuchungen an Compositen. Grana Palynologica, v. 2, p. 41-114, 1960.

Wodehouse, R.P. Pollen grains. New York: Mc Graw Hill, 1935. 